\title{
The effects of ram-pressure stripping on the internal kinematics of simulated spiral galaxies
}

\author{
T. Kronberger ${ }^{1,2}$, W. Kapferer ${ }^{1}$, S. Unterguggenberger ${ }^{1}$, S. Schindler ${ }^{1}$, and B. L. Ziegler $^{3}$ \\ 1 Institute for Astro- and Particle Physics, University of Innsbruck, Technikerstr. 25, 6020 Innsbruck, Austria \\ e-mail: Thomas.Kronberger@uibk.ac.at \\ 2 Max-Planck-Institut für Extraterrestrische Physik, Giessenbachstrasse, 85748 Garching bei München, Germany \\ ${ }^{3}$ European Southern Observatory, Karl-Schwarzschild-Strasse 2, 85748 Garching bei München, Germany
}

Received 11 January 2008 / Accepted 17 March 2008

\section{ABSTRACT}

\begin{abstract}
Aims. We investigate the influence of ram-pressure stripping on the internal gas kinematics of simulated spiral galaxies. Additional emphasis is put on the question of how the resulting distortions of the gaseous disc are visible in the rotation curve and/or the full 2D velocity field of galaxies at different redshifts.

Methods. A Milky-Way type disc galaxy is modelled in combined $N$-body/hydrodynamic simulations with prescriptions for cooling, star formation, stellar feedback, and galactic winds. This model galaxy moves through a constant density and temperature gas, which has parameters similar to the intra-cluster medium (ICM). We study five different configurations, in which the direction of the ram pressure on the gaseous disc varies from face-on to edge-on. Rotation curves (RCs) and 2D velocity fields of the gas are extracted from these simulations in a way that follows the procedure applied to observations of distant, small, and faint galaxies as closely as possible.

Results. We find that the appearance of distortions of the gaseous disc due to ram-pressure stripping depends on the direction of the acting ram pressure. In the case of face-on ram pressure, the distortions mainly appear in the outer parts of the galaxy in a very symmetric way. This distinction between undisturbed inner part and symmetrically disturbed outer regions is visible in both the rotation curve and the $2 \mathrm{D}$ velocity field. In contrast, in the case of edge-on ram pressure we find stronger distortions. In particular, a mismatch between kinematic centre and the centre of the stellar disc becomes observable. In the RC, there are basically two features visible. The $\mathrm{RC}$ is asymmetric as the kinematic centre does not coincide with the optical centre of the galaxy. Secondly, the outer parts of the RC are declining. The 2D velocity field also shows signatures of the interaction in the inner part of the disc. At angles smaller than $45^{\circ}$ between the ICM wind direction and the disc, the velocity field asymmetry increases significantly compared to larger angles. Compared to distortions caused by tidal interactions, the effects of ram-pressure stripping on the velocity field are relatively low in all cases and difficult to observe at intermediate redshift in seeing-limited observations.
\end{abstract}

Key words. galaxies: interactions - galaxies: intergalactic medium - galaxies: kinematics and dynamics - methods: numerical

\section{Introduction}

It has been pointed out ever since the late 1970's that there is an evolution of galaxy cluster members with redshift. The fraction of star-forming and post-star-forming systems, for example, has been proven to increase significantly with $z$ (Dressler et al. 1987, 1999). Consistently, Butcher \& Oemler (1978) reported a strong evolution from bluer to redder colours in cluster galaxies, detecting an excess of blue objects at $z=0.5$ with respect to lower redshift systems (this observational finding is usually referred to as "Butcher - Oemler effect"). Various physical mechanisms were proposed which act on the star formation activity and on the morphology of galaxies in clusters besides the hierarchical assembly of structures (e.g. ram-pressure stripping: Gunn \& Gott $1972-$ "harassment": e.g. Moore et al. 1998 - "strangulation" or "starvations": e.g. Larson et al. 1980). We still need to understand the specific importance of each of the proposed processes. For that purpose it is crucial to identify and disentangle different interactions, also at intermediate and high redshift. One possibility is to study the stellar population of the galaxies which, however, depends heavily on the current star formation activity. The time-dependent star formation encompasses several non-linear processes, which are partly poorly understood. Another method is to study the total gravitational potential of galaxies and its possible distortions due to interactions via their internal kinematics. Technically it became feasible to observe the full $2 \mathrm{D}$ velocity field (VF) of local galaxies in optical wavebands using integral field units (IFUs) such as SAURON (e.g. Ganda et al. 2006) or Fabry-Perot interferometry (e.g. Chemin et al. 2006; Garrido et al. 2002). For intermediate and high redshift galaxies, however, there are few observational studies of $2 \mathrm{D}$ velocity fields available and many studies still rely on rotation curves (RCs) from slit spectroscopy (e.g. Weiner et al. 2006; Moran et al. 2007; Böhm \& Ziegler 2007). To identify distortions and irregularities in the velocity fields is in both cases critical. A desirable goal would be to disentangle different interaction processes due to their influence on the velocity field. Numerical simulations offer a possibility to study individually the effects of the various processes. Kronberger et al. (2006, 2007) investigated in this way the effects of galaxy-galaxy mergers and of tidal interactions between galaxies on the internal kinematics of model galaxies. Those simulations were also used to identify possible observational biases in observations of the velocity field of distant galaxies (Kapferer et al. 2006; Kronberger et al. 2007). Although dependencies on the viewing angle and on the spatial resolution have been found, it was shown that tidal 
interactions mainly introduce nonaxisymmetric and nonbisymmetric features (see also Rubin et al. 1999).

Ram-pressure stripping is another important cluster-specific interaction process. The space between the galaxies in a galaxy cluster is filled with a hot $\left(\sim 10^{8} \mathrm{~K}\right)$, thin $\left(\sim 10^{3}\right.$ ions $\left./ \mathrm{m}^{3}\right)$ plasma, the so called intra-cluster medium (ICM). This plasma exerts a pressure on the inter-stellar medium (ISM), which can remove gas from the disc if the force due to ram-pressure stripping exceeds the restoring gravitational force of the galaxy. This mass loss due to ram-pressure stripping was modelled by many groups (e.g. Abadi et al. 1999; Vollmer et al. 2001; Roediger \& Hensler 2005; Quilis et al. 2000; Mori \& Burkert 2000; Toniazzo \& Schindler 2001; Schulz \& Struck 2001; and Jáchym et al. 2007). Recently also the effects of the external ram pressure on the star formation rate of the stripped galaxy were studied (Kapferer et al. 2008; Kronberger et al. 2008). Here we study the influence of ram-pressure stripping on the internal gas kinematics of simulated spiral galaxies.

This interaction mechanism has little influence on the stellar structure and could, therefore, be hidden in a galaxy that has a regular appearance when only direct imaging is used to search for interaction processes. However, it has a strong effect on the gas clouds within a galaxy disk disrupting its rotational motion, which can be traced by emission lines originating in these gas clouds. If spectra of observed galaxies have sufficient signal in the continuum, absorption lines can be used to also derive stellar rotation curves. Discrepancies between these and the gas motion are then strong indications of ram pressure. The velocity field of faint distant galaxies is in most cases measured only by emission lines (e.g. Ziegler et al. 2002; Bamford et al. 2005; Metevier et al. 2006) so that one needs a very thorough understanding of the effect of ram-pressure stripping.

Observable signatures of ram-pressure stripping were also studied numerically by Roediger \& Brüggen (2006, 2007) using Eulerian hydrodynamics. They focussed on the gas distribution in the disc and in the wake and thereby tried to deduce information on the interaction geometry. The velocity field in the wake has also been investigated. They have, however, not considered the velocity field in the disc in detail, as we do it in the present work. In a series of papers Vollmer et al. have used sticky particle simulations to model specific observed systems (Vollmer et al. 2000, 2003, 2006, 2008). They have tried to match observed HI velocity fields and gas distributions to infer information on the interaction scenario. Consequences of ram-pressure stripping for observable ICM properties are discussed in Schindler \& Diaferio (2008) and references therein. This present work goes beyond these previous studies by systematically investigating the effects of ram-pressure stripping observable with rotation curves from optical long-slit spectroscopy and $2 \mathrm{D}$ velocity fields at different redshifts.

We also study whether the deviations from a normal rotation caused by ram-pressure stripping have a different form than in the case of other interactions, so that they could be taken as an indicator of what mechanism may be acting on a galaxy. In the cluster and group environment it is expected that ram-pressure stripping is one of the main drivers for evolutionary effects on galaxies. It could be responsible for the global suppression of star formation in cluster galaxies (either through an initial star burst triggered by gas compression, as found e.g. by Kronberger et al. 2008; and Kapferer et al. 2008, or through the subsequent removal of gas from the disk). On the other hand, it cannot be responsible for structural changes needed for a complete transition from one galaxy type to another.
Table 1. Particle numbers and mass resolution used for the model galaxy.

\begin{tabular}{ccc}
\hline \hline & Particle number & $\begin{array}{c}\text { Mass resolution } \\
{\left[M_{\odot} / \text { particle }\right]}\end{array}$ \\
\hline Dark matter halo & 300000 & $3.5 \times 10^{6}$ \\
Disk collisionless & 200000 & $1.0 \times 10^{5}$ \\
Gas in disk & 200000 & $3.4 \times 10^{4}$ \\
ICM & 1000000 & $1.4 \times 10^{6}$ \\
\hline
\end{tabular}

The paper is organised as follows: in Sect. 2 we present the simulation setup used for this work. In Sect. 3 the extraction of the observable quantities (i.e. the rotation curves and the $2 \mathrm{D}$ velocity fields) are described. The results are presented in Sect. 4 and subsequently discussed with respect to previous observational and numerical work in Sect. 5. We end with a summary of the main conclusions in Sect. 6.

\section{Simulations}

The simulations were carried out with the $N$-body/SPH code GADGET-2 developed by V. Springel (see Springel 2005, for details). This code treats the gas of the galaxies and the ICM hydrodynamically via smoothed particle hydrodynamics (SPH, Gingold \& Monaghan 1977; Lucy 1977) while the collisionless dynamics of the dark matter and the stellar component is calculated using an $N$-body technique. Prescriptions for cooling, star formation (SF), stellar feedback, and galactic winds are included as described in Springel \& Hernquist (2003). The mass loss of the galaxy due to galactic winds, $\dot{M}_{w}$, is assumed to be proportional to the star formation rate $\Psi_{\mathrm{SFR}}$, i.e. $\dot{M}_{w}=\eta \Psi_{\mathrm{SFR}}$ with $\eta=2$, consistent with the observations of Martin (1999). Additionally, the wind contains a fixed fraction $\chi$ of the supernova energy, which is assumed to be $\chi=0.25$, as in Springel \& Hernquist (2003).

The initial conditions were built according to Springel et al. (2005), based on the analytical work of Mo et al. (1998). The model galaxy, which we use, represents a Milky-Way type spiral galaxy. The total mass of the model galaxy is $1.09 \times 10^{12} M_{\odot}$, where the initial total gas mass is $6.8 \times 10^{9} M_{\odot}$. The initial disc scale length is $r_{\mathrm{d}} \sim 3.3 \mathrm{kpc}$. Compared to previous work (Kapferer et al. 2005, 2006; Kronberger et al. 2006, 2007), where we used a similar sized galaxy, we have significantly increased the number of particles, increasing the mass resolution of the simulation. Important numerical quantities, such as the particle numbers, are summarised in Table 1. For the intra-cluster medium we use one million SPH particles, which we distribute homogeneously over a volume of $1 \mathrm{Mpc}^{3}$ with a mass density of $1 \times 10^{-28} \mathrm{~g} / \mathrm{cm}^{3}$ and a constant temperature of $3 \mathrm{keV}$ $\left(\sim 3.6 \times 10^{7} \mathrm{~K}\right)$. Such a simplified ICM distribution allows the effects of ram-pressure stripping to be studied in a clean way, i.e. with as little degeneracies of different effects as possible. Additional effects from varying density and temperature in the ambient medium will be investigated in an upcoming work.

Starting from these initial conditions we calculate the evolution of the model galaxy moving with a constant velocity of $1000 \mathrm{~km} \mathrm{~s}^{-1}$ through the ambient medium for $1 \mathrm{Gyr}$. We calculated five different interaction geometries, where we varied the angle between the ICM wind direction and the plane of the disc from $0^{\circ}$ to $90^{\circ}$. The edge-on and face-on stripping configurations are the two extreme cases where the perturbation acts either perpendicular to the galactic disc or purely in the plane of the gaseous disc. We study these two cases in detail. The other 
three simulations, where this angle is $23^{\circ}, 45^{\circ}$, and $68^{\circ}$, respectively, are used to study how the asymmetry of the velocity field depends on the ICM wind direction.

\section{Extraction of realistic rotation curves and 2D velocity fields}

In order to be able to compare our results to observations of distant, small, and faint galaxies we extract rotation curves and 2D velocity fields as described in Kronberger et al. (2006, 2007) in a way which closely resembles the observational approach (e.g. Ziegler et al. 2003). For the construction of realistic $2 \mathrm{D}$ velocity fields, we project all gas particles onto a Cartesian, equally spaced grid. The spacing is chosen such that the spatial resolution corresponds to the angular resolution of current state-ofthe-art observations. We adopt an angular resolution typical for IFU or FPI observations, namely 0.4" (e.g. Chemin et al. 2006). The angular resolution of SAURON, for example, would be $0.3^{\prime \prime}$ or $0.9^{\prime \prime}$. We calculate the physical resolution according to the given angular resolution for a particular redshift using the concordance cosmological model. The velocity field of the galaxy is binned using this spatial resolution.

For observations of galaxies at intermediate and high redshift seeing plays a crucial role, as it typically exceeds the angular resolution of the instrument. To simulate seeing effects on the velocity-field measurements we apply a convolution with a Gaussian point spread function. A value of $0.8^{\prime \prime}$ for the $F W H M$ of the Gaussian seeing was adopted, which is a typical value for ground based observations (see e.g. Jäger et al. 2004) and is reasonable for the achieved spectral resolution (and a slit width of $\left.1^{\prime \prime}\right)$.

From this velocity field we can further extract a rotation curve by modelling a slit which is placed over the major axis of the galaxy with a typical slit width of $1^{\prime \prime}$, as detailed in Kronberger et al. (2006).

\section{Results}

In the following we investigate observable projections of the full $3 \mathrm{D}$ velocity field of the simulation, i.e. the rotation curves and the $2 \mathrm{D}$ velocity fields. Special emphasis is put on the question how the given distortions of the velocity field appear at intermediate redshift.

We quantify the degree of asymmetry of the RC shape, following an approach used in Dale et al. (2001) and subsequently for simulated galaxies in Kronberger et al. (2006). The area between the kinematically folded approaching and receding halves is divided by the average area under the RC:

$$
\begin{aligned}
\text { Asymmetry }= & \sum \frac{\| V(r)|-| V(-r)||}{\sqrt{\sigma^{2}(r)+\sigma^{2}(-r)}} \\
& \times\left[\frac{1}{2} \sum \frac{|V(r)|+|V(-r)|}{\sqrt{\sigma^{2}(r)+\sigma^{2}(-r)}}\right]^{-1} .
\end{aligned}
$$

Here $V(r)$ is the velocity and $\sigma(r)$ is the uncertainty of the rotational velocity at position $r$. As in Kronberger et al. (2006) we adopt for $\sigma(r)$ a value of $20 \mathrm{~km} \mathrm{~s}^{-1}$, which is typically the minimum uncertainty in observations of distant galaxies due to the limited spectral resolution.

In order to quantify the distortions and to interpret the partly complex structures in the $2 \mathrm{D}$ velocity fields we use as in Kronberger et al. (2007) the kinemetry package of
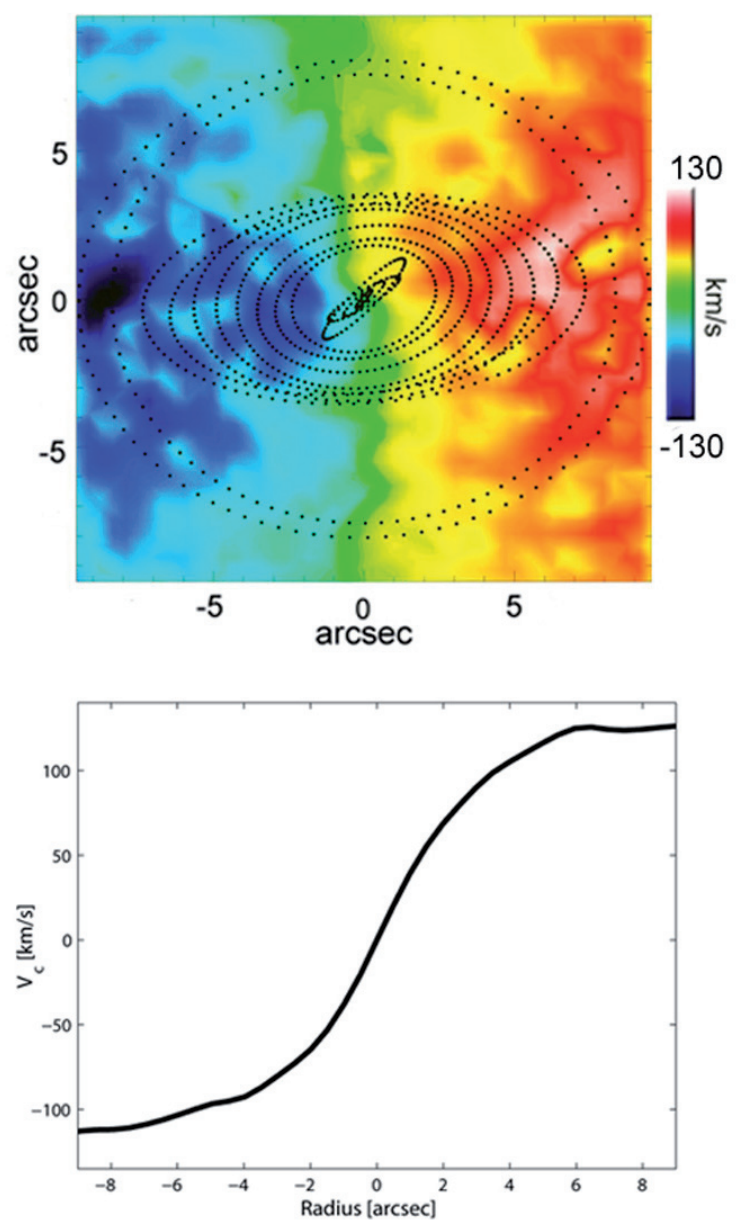

Fig. 1. 2D velocity field and long-slit rotation curve of an undisturbed model galaxy as seen at redshift $z=0.1$. The figure of the $2 \mathrm{D}$ velocity field is taken from Kronberger et al. (2007). Overlayed as contours are the best fitting ellipses from the kinemetry analysis.

Krajnović et al. (2006). This method is based on the assumption that the mean velocity along best fitting ellipses is reproduced by a simple cosine law, i.e.

$V(a, \Psi)=V_{0}+V_{\mathrm{c}}(a) \cos \Psi$,

where $a$ is the length of semi-major axis of the ellipse, $\Psi$ for discs is the azimuthal angle measured from the major axis in the plane of the galaxy. The position angle $\Gamma$ and the axial ratio $(q=$ $b / a)$ of the ellipses are calculated as a function of radius from the galactic centre. Deviations from the cosine law are measured using an harmonic expansion along the ellipses, i.e.

$V(a, \Psi)=\sum_{n=1}^{N} k_{n}(a) \cos \left[n\left(\Psi-\phi_{n}(a)\right)\right]$,

where $\phi$ is the phase coefficient. Higher order Fourier terms and radial changes of $\Gamma$ or $q$ quantize deviations in the velocity field from a simple rotation. Sometimes a simple sine correction for the inclination of the disc is applied in observations. As this correction simply alters the absolute value of the velocities, we do not apply it to our model velocity fields. All VFs were extracted at the same inclination $i=35^{\circ}$.

In Fig. 1 we present the $2 \mathrm{D}$ velocity field and a long-slit RC from an undisturbed model galaxy as seen at redshift $z=0.1$. For the appearance of the VF at different redshifts we refer to Kronberger et al. (2007). 


\subsection{Rotation curves of ram-pressure stripped galaxies}

First we study the RC of a model galaxy moving face-on through the ambient medium. After 100 Myr of interaction a clear stripping radius is visible, which stays roughly constant over time and agrees with analytical estimates (Kronberger et al. 2008). The stripping radius in ram-pressure affected galaxies is defined, as the distance from the galactic centre, outside which the interstellar medium (ISM) cannot be prevented from being stripped by the galactic gravitational potential. We extract rotation curves from the galaxy before the clear emergence of a stripping radius, i.e. in the early phases of stripping (after 50 Myr), and thereafter (after $\sim 100 \mathrm{Myr}$ ). The RC of the isolated model galaxy has the typical shape, rising in the inner and flattening in the outer parts. After $50 \mathrm{Myr}$ of acting ram pressure first effects of the external pressure are visible in the distribution of the gas. The strength of the disturbances depends on the local gravitational potential. They are strongest at large galactic radii, where the restoring force acting against ram pressure is weakest. Whether these distortions are visible in the rotation curve depends on the radius to which the $\mathrm{RC}$ can be measured. We assume that the rotational velocity can be determined accurately out to 4 times the radial disc scale length of the galaxy. This is a typical value obtainable at intermediate redshift (e.g. Ziegler et al. 2003). In the top panel of Fig. 2 we present the corresponding RC, which does not show strong peculiarities (asymmetry measure $\sim 6 \%$ ).

Local measurements extend to much larger radii. We consider the case where the rotational velocity can be measured out to 6 times the radial disc scale length. Already after $50 \mathrm{Myr}$, the gas at such large radii gets a dominant velocity component antiparallel to the galaxies' direction of movement, i.e. away from the galactic disc. How this change of the velocity vector in the stripping region translates to a change in the $\mathrm{RC}$ of the galaxy is shown in the lower panel of Fig. 2. In the outer parts of the $\mathrm{RC}$, a turn-over of the rotational velocity on both sides to higher negative values can be observed. This is a consequence of gas moving away from the galactic disc due to the ram-pressure. The asymmetry measure of this disturbed RC is $\sim 22 \%$, which is low compared to distortions caused by tidal interactions (see Kronberger et al. 2007). Note that the "direction" of the distortion depends on the movement of the galaxy with respect to the observer. If the galaxy was moving away from the observer, the rotational velocities in the outer parts of the $\mathrm{RC}$ would go to higher positive values.

After 100 Myr the stripping radius at $r \sim 12 \mathrm{kpc}$ is clearly visible. Hence also the RC distortion is now visible in this region of the disc, and also observable at intermediate redshift. The RC for this time step is presented in Fig. 5 (top panel). There is a dependence of the RC shape on the inclination of the galactic disc but the important characteristics of the RC remain observable for most inclinations. These characteristic features in the outer parts of the RC are less prominent if the galaxy is observed almost edge-on. There the gas velocity is dominated by a component perpendicular to the galactic disc due to the acceleration by the ICM wind. If the galaxy is seen edge-on, only the altered disc velocity component is observed. The dependence on the viewing angle is not as strong as for tidal interactions (see Kronberger et al. 2006) as ram-pressure stripping is an axisymmetric distortion. The characteristic features are observable for all viewing angles.

Next we investigate the RC of a model galaxy flying edge-on through the ambient medium. In this case the perturbation acts in the plane of the disc. The gaseous disc gets strongly compressed, which alters the radial pressure gradients and hence the
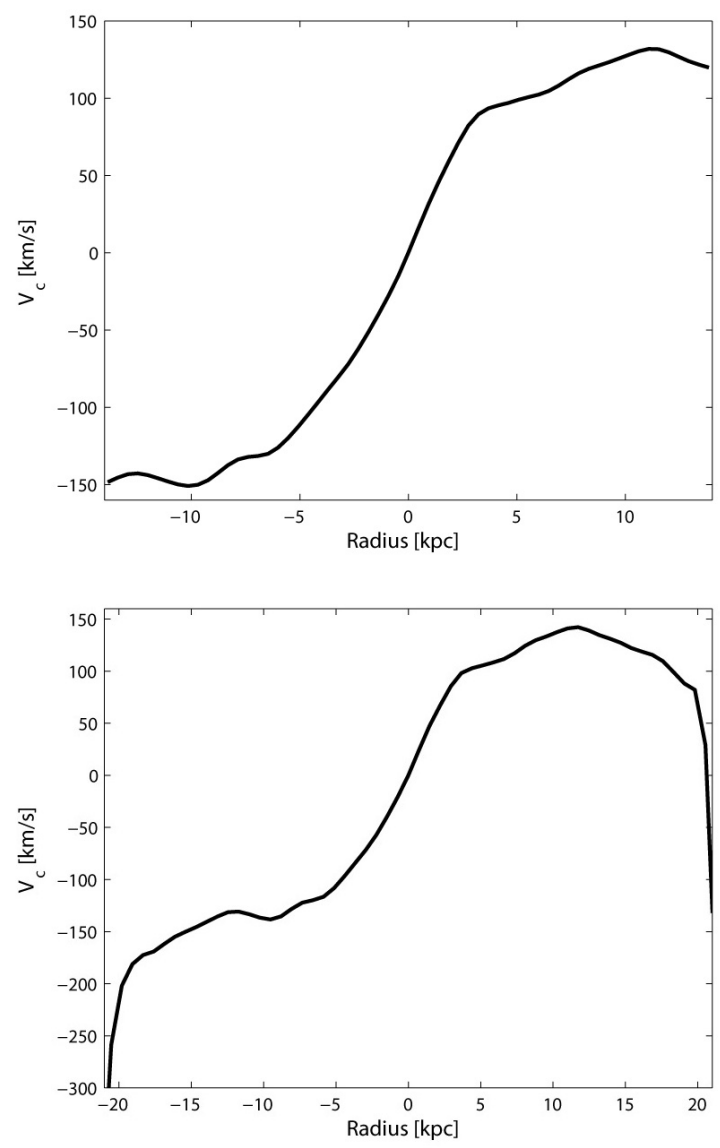

Fig. 2. Rotation curve of a model galaxy after $50 \mathrm{Myr}$ of ram pressure acting face-on. The RC is assumed to be observable out to 4 (top panel) and 6 (lower panel) times the radial disc scale length, respectively. In the top panel, the $\mathrm{RC}$ is similar to the initial undisturbed configuration. In the lower panel signatures of ram-pressure stripping are clearly visible in the outer parts. Note that the "direction" of the distortion depends on the movement of the galaxy with respect to the observer. If the galaxy was moving away from the observer, the rotational velocities in the outer parts of the RC would go to higher positive values.

rotational velocity of the gas. We find stronger distortions than in the face-on case, especially a mismatch between kinematic centre and the centre of the stellar disc is observable. The offset of the gas kinematic centre is $\approx 2 \mathrm{kpc}$ after $500 \mathrm{Myr}$ of ram pressure acting. In Fig. 3 we show the contours of the gas distribution overlayed on the smoothed stellar distribution after $500 \mathrm{Myr}$ of ram-pressure acting (we chose this evolved time step, compared to the face-on case, as the distortions are best visible there. Before, the distortions are qualitatively the same, but less prominent). In contrast to the face-on stripping, there is no distinct centre or symmetry visible in the gas distribution anymore. In the rotation curve, there are basically two features visible. As the kinematic centre does not coincide with the optical centre of the galaxy anymore, the RC is slightly more asymmetric than in the case of face-on ram pressure (asymmetry measure $\sim 30 \%$ ). Secondly, the $\mathrm{RC}$ is declining in the outer part on both sides. Note that this feature is in this case only visible in the RC of the gas, as only this component is affected by the ram pressure of the ambient medium. The collisionless components of the galaxy (i.e. the stars and the dark matter) are not or due to the changing gas density distribution only mildly affected. If a declining RC is also observed in the stellar RC, a tidal interaction affecting also the stars and the dark matter is most likely the cause. There is a 


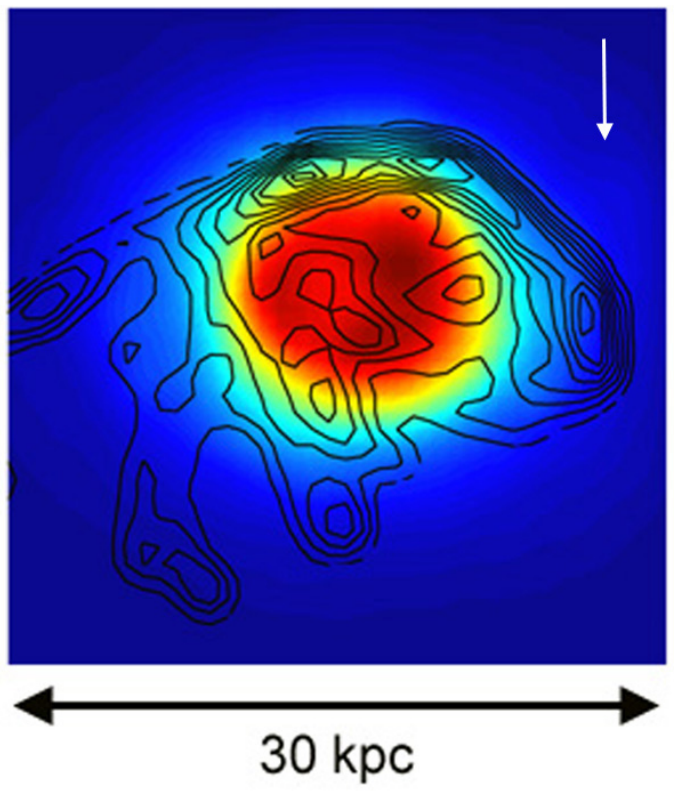

Fig. 3. The gas distribution is overlayed as contours on the smoothed stellar distribution after $500 \mathrm{Myr}$ of ram pressure acting edge-on. The white arrow in the top right corner of the figure indicates the ICM wind direction. The galaxy rotates counter-clockwise.

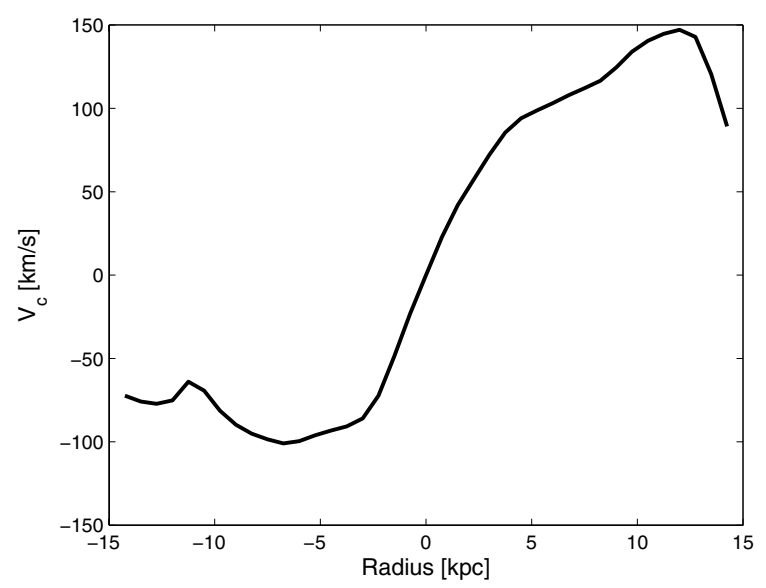

Fig. 4. Rotation curve of a model galaxy after $500 \mathrm{Myr}$ of ram pressure acting edge-on.

dependence on the viewing angle and the inclination of the disc, not strongly affecting, however, the main feature of the disturbed $\mathrm{RC}$, namely the declining outer parts.

For better visual judgement of the asymmetry of the RC, both its sides are sometimes plotted on one side. In Fig. 5 we present the RCs in such a way for the model galaxy after $100 \mathrm{Myr}$ of face-on ram pressure and after $500 \mathrm{Myr}$ of edge-on ram pressure, respectively.

In both cases, edge-on and face-on, the long-slit RC cannot be used to estimate parameters for a Tully-Fisher study. The kinematics of the gas in the galaxy is affected by rampressure stripping. The unaffected stellar kinematics could be used instead to estimate the maximum rotational velocity $V_{\max }$. However, the luminosity of the galaxy is also affected by rampressure stripping. In a recent work, we found an enhancement of the star-formation rate due to ram pressure (Kronberger et al. 2008). A subsequent brightening of the ram-pressure affected galaxy can cause an increased scatter in the Tully-Fisher relation.
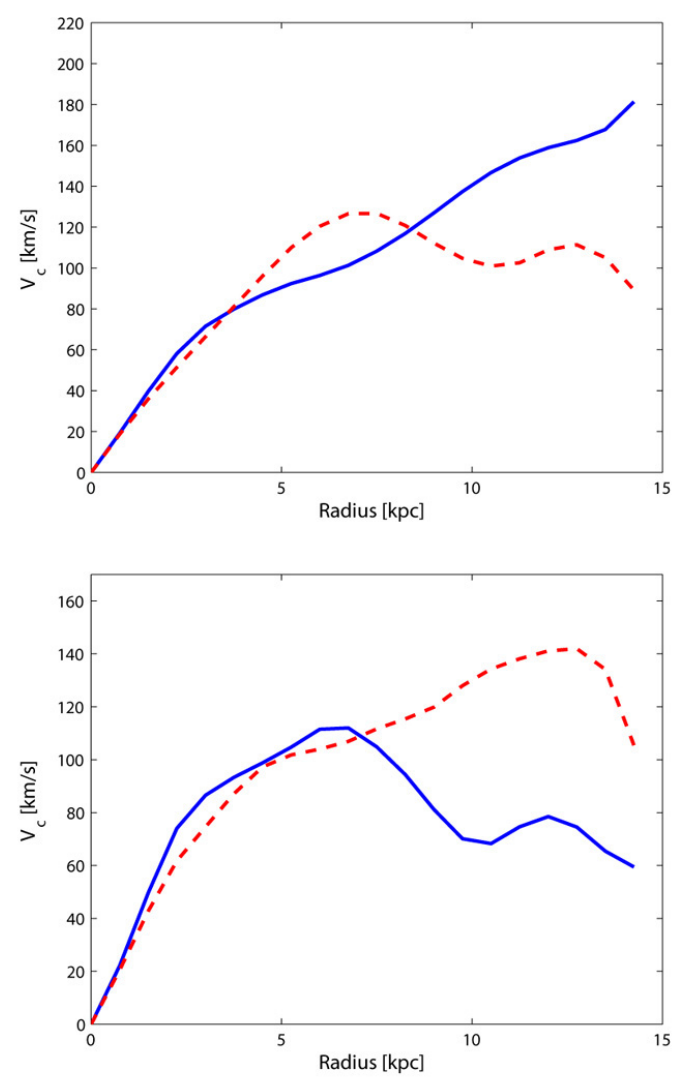

Fig. 5. Rotation curve of a model galaxy after $100 \mathrm{Myr}$ of ram pressure acting face-on (top panel) and after $500 \mathrm{Myr}$ of ram pressure acting edge-on (bottom panel). Both sides of the RC are plotted on one side for better judgement of the asymmetry. The blue-shifted part of the RC is plotted as blue solid line, while the redshifted part is plotted as dashed red line.

In Fig. 6 we study how the asymmetry of the VF depends on the angle between the ICM wind direction and the plane of the disc. The asymmetry measure depends on the viewing angle and the inclination, so we averaged over three different linesof-sight and two observed disc inclinations. A clear step in the asymmetry measure can be seen at $45^{\circ}$, where the asymmetry increases significantly. The scatter, however, increases as well, which makes it difficult to distinguish further between different ICM wind directions with an observed VF asymmetry. A high VF asymmetry is nevertheless a good hint, for a small angle between the ICM wind direction and the plane of the disc.

\section{2. $2 D$ velocity fields of ram-pressure stripped galaxies}

With the help of $2 \mathrm{D}$ velocity fields the nature of the interaction becomes more accessible than with simple rotation curves from long-slit spectroscopy. Quantitative analyses on the kinematic axis, the kinematic centre and more detailed analyses such as harmonic expansion, which is, for example, used by the kinemetry package of Krajnović et al. (2006), offer additional possibilities to identify distortions in a VF. We have already applied the kinemetry method to tidally disturbed simulated systems in Kronberger et al. (2007).

In Fig. 7 we show such a 2D velocity field of a face-on ram-pressure affected galaxy after $250 \mathrm{Myr}$ of ram pressure acting. (The distortions at this time step are qualitatively equal to those present after $100 \mathrm{Myr}$, but more prominent. Therefore we 


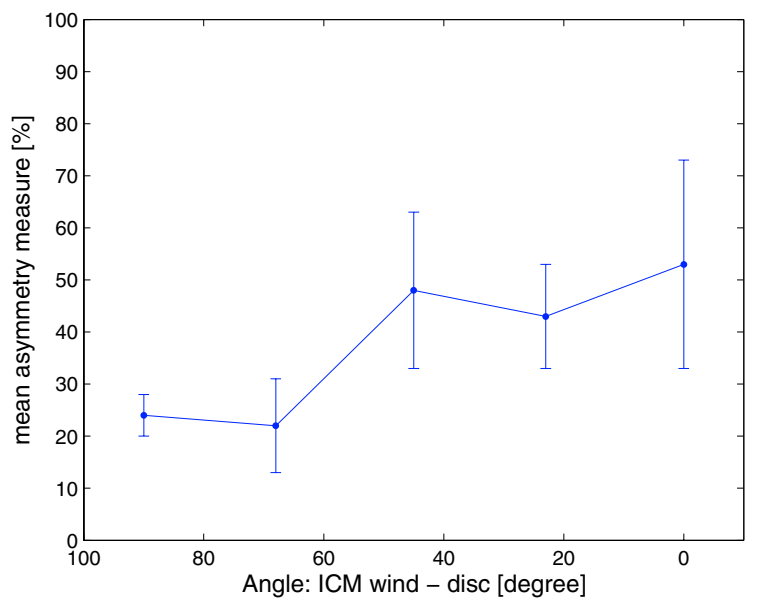

Fig. 6. Asymmetry measure as a function of the angle between the disc and the ICM wind direction $\left(0^{\circ}\right.$ is edge-on and $90^{\circ}$ is face-on ram pressure). The asymmetry measure was averaged over several viewing angles. The error bars indicate the standard deviation of the mean.

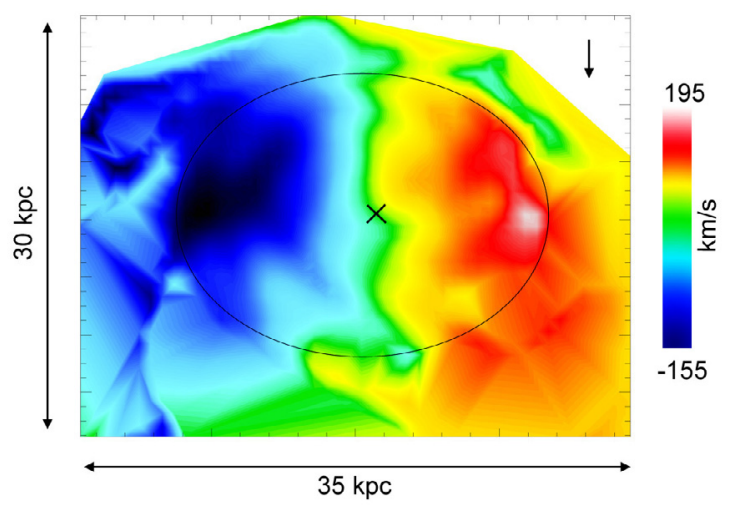

Fig. 7. 2D velocity field of a face-on ram-pressure affected galaxy after $250 \mathrm{Myr}$ of ram pressure acting. The almost undisturbed inner part of the velocity field is highlighted by an ellipse, whose semi-major axis roughly corresponds to the stripping radius $(\sim 12 \mathrm{kpc}$ as estimated in Kronberger et al. 2008). The centre of the stellar disc is indicated by the black cross. The black arrow in the top right corner indicates the projected ICM wind direction. Note that the ICM wind acts perpendicular to the plane of the disc.

chose this particular time step, without loss of generality). The distortions are not as severe as those caused by tidal interactions, which were presented in Kronberger et al. (2007). An almost undisturbed inner part of the velocity field is clearly visible, which we indicated with an ellipse. The semi-major axis of this ellipse corresponds roughly to the stripping radius $(12 \mathrm{kpc})$ of this model system as estimated in Kronberger et al. (2008). Beyond this radius the VF appears disturbed. This picture of an undisturbed inner part and a severely affected outer part beyond the stripping radius also agrees with the rotation curve of the system presented in the previous section (cf. Fig. 2). As for the rotation curve, the VF appearance depends on the inclination. The characteristic features become less prominent when the galaxy is observed close to edge-on. In Fig. 8 we study the appearance of this velocity field when put at intermediate redshift (the same investigation as performed in Kronberger et al. (2007) for tidally disturbed velocity fields).

The VF appears smooth and widely undisturbed at $z=0.4$, as the irregularities are smeared out, mainly due to seeing (therefore the use of integral field spectrographs in combination with
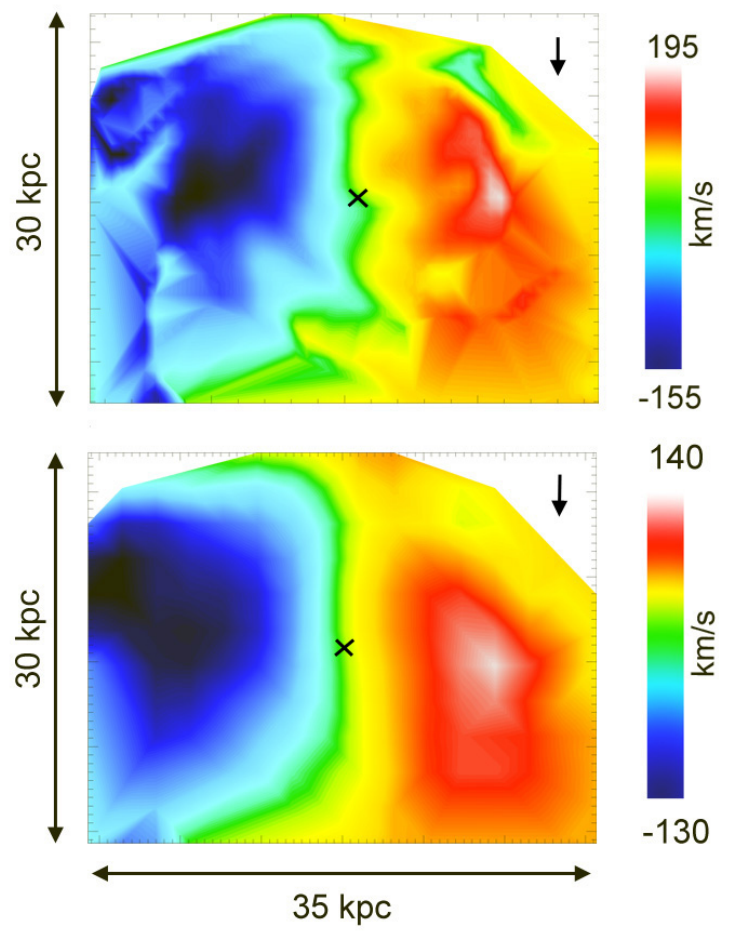

Fig. 8. The $2 \mathrm{D}$ velocity field of a face-on ram-pressure affected galaxy after $250 \mathrm{Myr}$ of ram pressure acting as seen at redhift $z=0.1$ (top panel) and $z=0.4$ (lower panel), respectively. The centre of the stellar disc is indicated by the black cross. The black arrow in the top right corner of each panel indicates the projected ICM wind direction. Note that the ICM wind acts perpendicular to the plane of the disc.

adaptive optics is desirable). The radial profiles of the kinemetric properties, which we calculated using the kinemetry programme of Krajnović et al. (2006), are presented in Fig. 9 for the model galaxy after $250 \mathrm{Myr}$ of face-on ram pressure acting. The position angle $\Gamma$ and the flattening $q$ of the best fitting ellipses as well as the first and the fifth order Fourier terms $k_{1}$ and $k_{5}$ are plotted as a function of radius for redshift $z=0.1$ (top) and $z=0.4$ (bottom). In the radial behaviour of these quantities the undisturbed inner part of the velocity field and the distorted outer part are visible. At a radius of $\sim 5.5^{\prime \prime}$ (at $z=0.1$, corresponding to $\sim 10 \mathrm{kpc}$ ) a clear increase in the ratio of $k_{5}$ and $k_{1}$ can be seen. This ratio represents complex, kinematically separate components in the velocity field. At the same radius the flattening of the ellipses and the first order Fourier term $k_{1}$ also show significant variations, which are, however, lower than in the case of tidally induced distortions (cf. corresponding figures in Kronberger et al. 2007). At a redshift of $z=0.4$, the variations of the radial kinemetric properties get less prominent. A clear identification of ram pressure affected galaxies is especially difficult as at such redshifts often only the inner part of the velocity field is observable. In the outer regions of the disc some irregularities in the velocity field remain visible also at intermediate redshift. They are observable as increase in the ratio of $k_{5}$ and $k_{1}$.

In Fig. 10 we present the 2D velocity field of an edge-on rampressure affected galaxy after $400 \mathrm{Myr}$ of ram pressure acting as seen at redhift $z=0.1$ (top panel) and $z=0.4$ (lower panel), respectively. The mismatch between kinematic centre and the centre of the stellar disc is the strongest effect of edge-on ram pressure. Already by visual assessment of the VF presented in Fig. 10 several distortions are visible also in the inner part of the VF. These irregularities are, however, completely smeared out at 

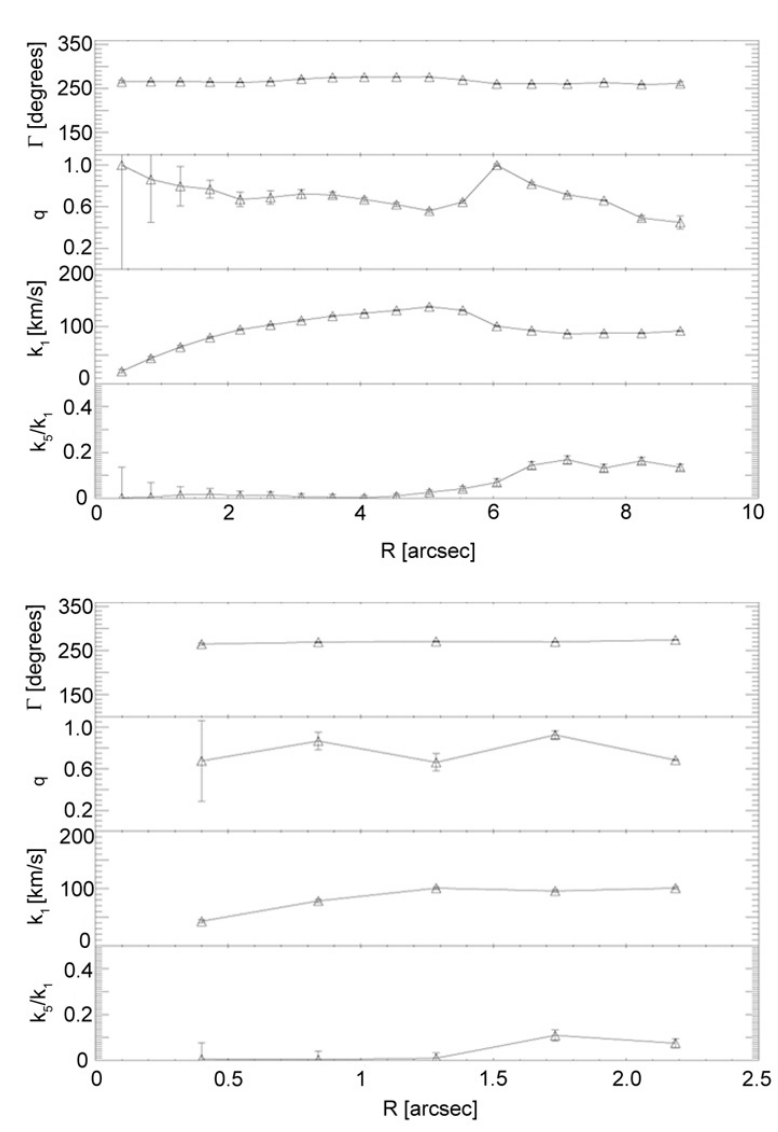

Fig. 9. Radial profiles of the kinemetric properties, calculated using the kinemetry programme for the model galaxy after $250 \mathrm{Myr}$ of face-on ram pressure acting, for redshift $z=0.1$ (top) and $z=0.4$ (bottom). The position angle $\Gamma$ and the flattening $q$ of the best fitting ellipses as well as the first and the fifth order Fourier terms $k_{1}$ and $k_{5}$ are plotted as a function of radius.

redshift $z=0.4$. Also the radial profiles of the kinemetric properties, presented in Fig. 11 reflect this behaviour. At $z=0.1$ variations of the flattening parameter $q$ and of the ratio of $k_{5}$ and $k_{1}$ are already visible at smaller radii. The amplitude of these variations is however small. To study the spatial distribution of the cold gas seems therefore to be still the best way to identify ram pressure acting in a galaxy. The influence on the velocity field is relatively low, as the rotational velocity depends mostly on the gradients of the gravitational potential and the gas accounts only for a small mass fraction.

\section{Discussion}

Ram-pressure stripping has been modelled numerically using different techniques. All these approaches use some simplifications due to the complexity of the multi-phase, multi-scale gas physics present in the inter-stellar medium of galaxies. One method is to treat the ISM hydrodynamically either with an Eulerian code (e.g. Roediger \& Brüggen 2007) or using SPH (e.g. Jáchym et al. 2007). SPH has the advantage that it can be easily combined with collisionless $N$-body simulations for the dark matter and stellar component. In the setup used for this work the collisionless component and the gas are treated fully self-consistently. An alternative method is to model the ISM with particles which can have inelastic collisions, so-called "sticky particles" (e.g. Vollmer et al. 2006). The ICM ram pressure
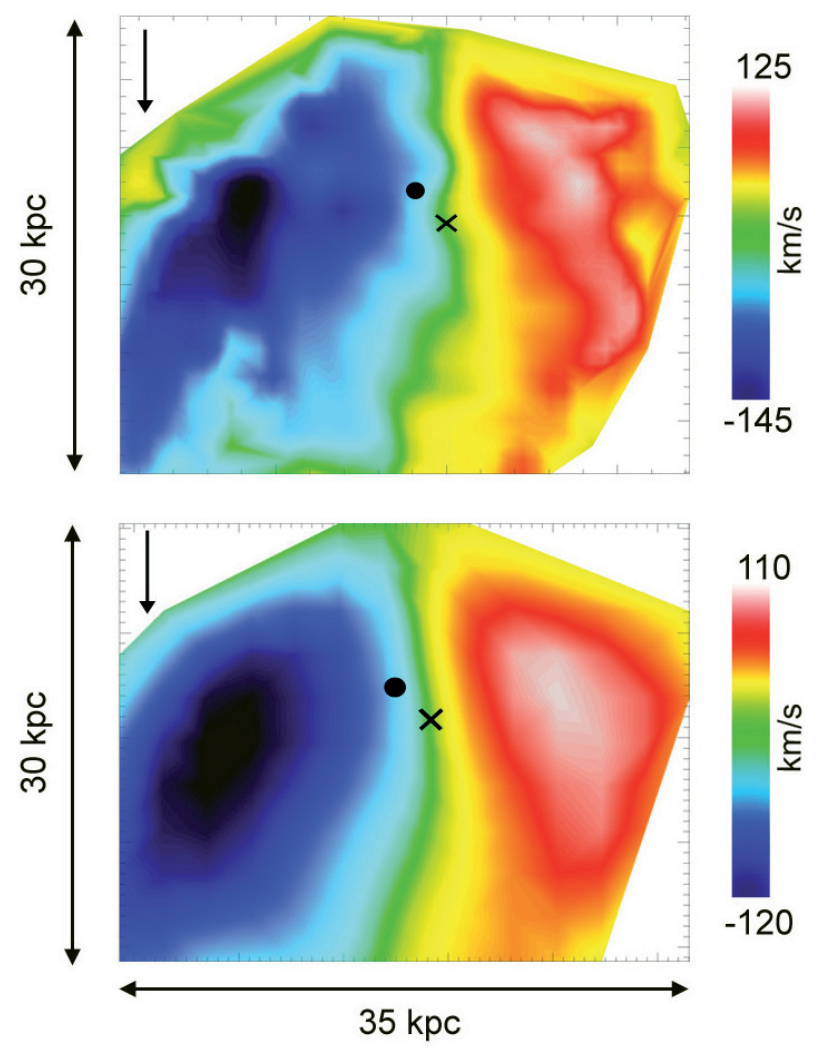

Fig. 10. 2D velocity field of an edge-on ram-pressure affected galaxy after $400 \mathrm{Myr}$ of ram pressure acting as seen at redhift $z=0.1$ (top panel) and $z=0.4$ (lower panel), respectively. The black arrow in the top left of each panel indicates the ICM wind direction and the cross and the circle indicate the kinematic and stellar disc centre, respectively.

is then modelled as additional acceleration on particles, which are not shielded by others. All these methods have their advantages and restrictions. If the ISM is considered as continuous fluid the hydrodynamic prescription yields a better model than sticky particles. The latter method is more suitable for noncontinuous systems (e.g. Vollmer et al. 2006). The SPH method has intrinsic shortcomings in the resolution of instabilities and turbulence (e.g. Agertz et al. 2007). In systems affected by rampressure stripping shear flows are present, which can lead to the development of Kelvin-Helmholtz instabilities (e.g. Roediger et al. 2006). These are not resolved in our setup. The KelvinHelmholtz instabilities affect mostly the stripped material in the wake, where the shear flow is strong and the gravitational force is weak (Roediger et al. 2006). The effect for the bulk motion of the gas in the plane of the disc is probably small. A similar study as the one presented in the present work should be carried out with a Eulerian code in a future work to investigate the importance of hydrodynamic instabilities in this issue. The huge advantage of our setup is that we treat gas, dark matter and the stellar component fully self-consistently.

Some of the results presented in this work were also found in previous studies. Roediger \& Brüggen (2007), for example, found that the gas distribution of ram-pressure affected galaxies is more asymmetric for edge-on ram pressure. Consistently we find this trend also in the velocity field. The mismatch between the kinematic and the stellar disc centre is caused by two effects. Ram pressure affects the whole disc. The gas gets dislocated where the restoring gravitational force is too low. This ram-pressure pushing (Roediger \& Brüggen 2007) leads also to a central density enhancement and consequently to an 

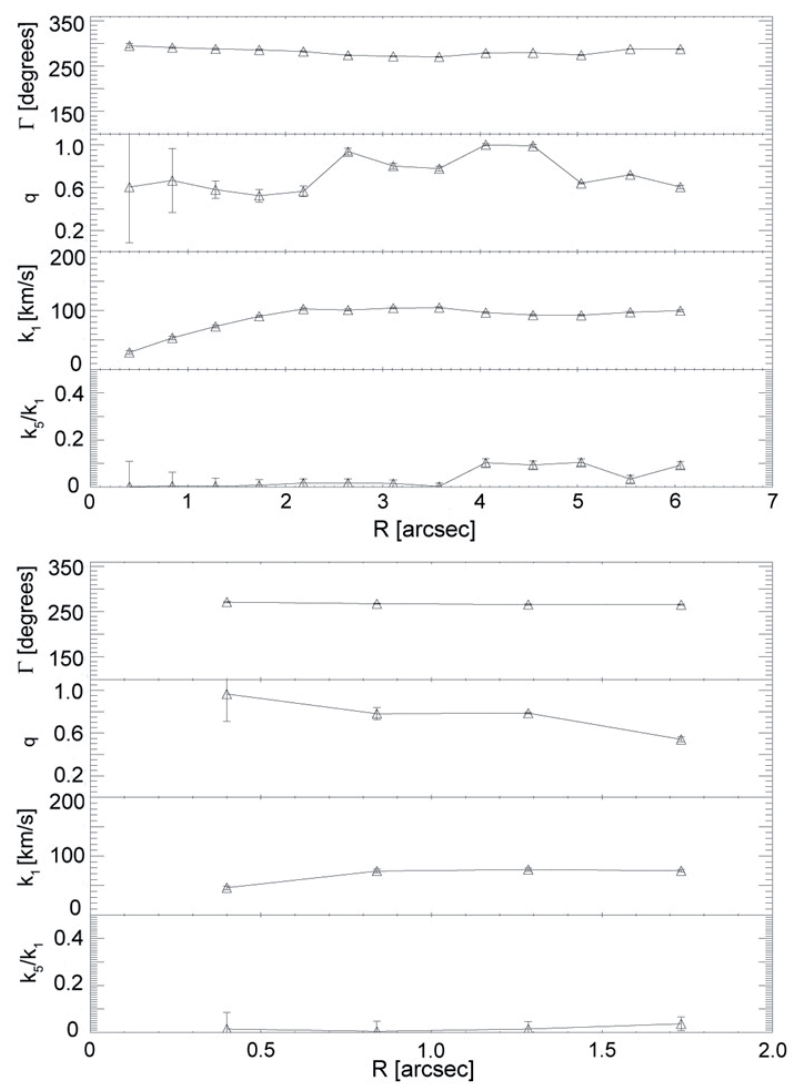

Fig. 11. Radial profiles of the kinemetric properties, calculated using the kinemetry programme for the model galaxy after 400 Myr of edgeon ram pressure acting, for redshift $z=0.1$ (top) and $z=0.4$ (bottom). The position angle $\Gamma$ and the flattening $q$ of the best fitting ellipses as well as the first and the fifth order Fourier terms $k_{1}$ and $k_{5}$ are plotted as a function of radius.

increased central star formation (Kronberger et al. 2008; Vollmer et al. 2001). Within our model this enhanced star formation is self-consistently treated and induces an increased galactic wind. These feedback mechanisms, which do affect the gas kinematics due to modified density and pressure gradients, were neglected in previous studies and are one of the strengths of the present work. With an offset of $\approx 2 \mathrm{kpc}$, the effect is, however, relatively small.

In a series of papers Vollmer et al. use the velocity field information to fit models of ram-pressure affected galaxies to HI observations of nearby cluster galaxies (e.g. Vollmer et al. 2008). They identify characteristic features in the VFs of the observed systems and study in which configuration the model can reproduce these features. In Vollmer et al. (2008) the velocity field of an almost edge-on stripped galaxy is shown. The VF is quite regular as the galaxy is in an early phase of stripping. Thus a direct comparison to our models is difficult. Even for edge-on stripping the VF distortions are moderate in our models. This is also true for the simulations of Vollmer et al. (2008) (compare e.g. their Fig. 20). Also in Vollmer et al. (2006) the VF appears rather regular. The VF of NGC 4569 presented in Vollmer et al. (2004) shows an asymmetric velocity field with an angle between orbital and disc plane of $35^{\circ}$. This is consistent with our finding that the velocity field becomes asymmetric for angles $<45^{\circ}$.

More detailed comparisons with observed optical velocity fields and rotation curves from the sample of Ziegler et al. (2007) will be subject of an upcoming work.

\section{Summary and conclusions}

We have investigated 2D velocity fields and rotation curves of the gas in ram-pressure affected spiral galaxies using $\mathrm{N}$-body/SPH simulations.

- We found that the appearance of distortions of the gaseous disc due to ram-pressure stripping depends on the direction of the acting ram-pressure. In our investigation we studied the two extreme cases of face-on and edge-on ram pressure. In both cases, the velocity fields of the ram-pressure affected galaxies show characteristic features.

- In general, the distortions of the gas velocity field are significantly lower than in the case of distortions induced by tidal interactions. The reason is that the rotational velocity depends mostly on the gravitational potential and the gas accounts only for a small mass fraction.

- In the case of face-on acting ram pressure distortions appear mainly in the outer parts of the galaxy. The separation between undisturbed inner and symmetrically disturbed outer part is visible in both the rotation curve and the $2 \mathrm{D}$ velocity field.

- In the case of ram pressure acting edge-on we find a significant mismatch between kinematic centre and the centre of the stellar disc. The rotation curve is asymmetric and the outer parts of the $\mathrm{RC}$ are declining. The $2 \mathrm{D}$ velocity field shows signatures of the interaction also in the inner part of the disc.

- At an angle of $45^{\circ}$ between the ICM wind direction and the disc, the velocity field asymmetry increases significantly with respect to smaller angles. The scatter is, however, increasing towards the edge-on wind too. Therefore it seems difficult to distinguish further between different ICM wind directions purely with the help of the VF asymmetry.

- The collisionless stellar disc is not affected by ram pressure and hence shows no disturbed kinematics. The characteristic features in the gas kinematics presented here, together with an absence of distortions in the stellar kinematics are therefore a strong indication for the presence of ram-pressure stripping acting on a cluster galaxy.

Acknowledgements. We thank the anonymous referee for fruitful comments which helped to improve the quality of the paper. The authors thank Volker Springel for providing them with GADGET2 and his initialconditions generator and Davor Krajnović for his Kinemetry software. Thomas Kronberger is a recipient of a DOC fellowship of the Austrian Academy of Sciences. The authors further acknowledge the UniInfrastrukturprogramm des BMWF Forschungsprojekt Konsortium Hochleistungsrechnen, the ESO Mobilitätsstipendien des BMWF (Austria), the Austrian Science Foundation (FWF) through grants P18523-N16 and P19300-N16, the German Science Foundation (DFG) through Grant number $\mathrm{Zi}$ 663/6-1, the Volkswagen Foundation (I/76 520), and the Tiroler Wissenschaftsfonds (Gefördert aus Mitteln des vom Land Tirol eingerichteten Wissenschaftsfonds). We further thank Chiara Ferrari for fruitful discussion.

\section{References}

Abadi, M. G., Moore, B., \& Bower, R. G. 1999, MNRAS, 308, 947

Agertz, O., Moore, B., Stadel, J., et al. 2007, MNRAS, 380, 963

Bamford, S. P., Milvang-Jensen, B., Aragón-Salamanca, A., \& Simard, L. 2005, MNRAS, 361, 109

Böhm, A., \& Ziegler, B. L. 2007, ApJ, 668, 846

Butcher, H., \& Oemler, A. Jr. 1978, ApJ, 219, 18

Chemin, L., Balkowski, C., Cayatte, V., et al. 2006, MNRAS, 366, 812

Dale, D. A., Giovanelli, R., Haynes, M. P., Hardy, E., \& Campusano, L. E. 2001, AJ, 121, 1886

Dressler, A. 1987, in Nearly Normal Galaxies: From the Planck Time to the Present, ed. S. Faber (New York: Springer), 276

Dressler, A., Smail, I., Poggianti, B., et al. 1999, ApJS, 122, 51 
Ganda, K., Falcón-Barroso, J., Peletier, R. F., et al. 2006, MNRAS, 367, 46 Garrido, O., Marcelin, M., Amram, P., \& Boulesteix, J. 2002, A\&A, 387, 821 Gingold, R. A., \& Monaghan, J. J. 1977, MNRAS, 181, 375

Gunn, J. E., \& Gott, J. R. III 1972, ApJ 176, 1

Jáchym, P., Palouš, J., Köppen, J., \& Combes, F. 2007, A\&A, 472, 5

Jäger, K., Ziegler, B. L., Böhm, A., et al. 2004, A\&A, 422, 907

Kapferer, W., Knapp, A., Schindler, S., Kimeswenger, S., \& van Kampen, E. 2005, A\&A, 438, 87

Kapferer, W., Kronberger, T., Schindler, S., Ziegler, B. L., \& Böhm A. 2006, A\&A, 446, 847

Kapferer, W., et al. 2008, A\&A, submitted

Krajnović, D., Cappellari, M., de Zeeuw, P. T., \& Copin, Y. 2006, MNRAS, 366, 787

Kronberger, T., Kapferer, W., Schindler, S., et al. 2006, A\&A, 458, 69

Kronberger, T., Kapferer, W., Schindler, S., \& Ziegler, B. L. 2007, A\&A, 473, 761

Kronberger, T., Kepferer, W., Ferrari, C., Unterguggenberger, S., \& Schindler, S. 2008, A\&A, 481, 337

Larson, R. B., Tinsley, B. M., \& Caldwell, C. N. 1980, ApJ, 237, 692

Lucy, L. B. 1977, AJ, 82, 1013

Martin, C. L. 1999, ApJ, 513, 156

Metevier, A. J., Koo, D. C., Simard, L., \& Phillips, A. C. 2006, ApJ, 643, 764

Mo, H. J., Mao, S., \& White, S. D. M. 1998, MNRAS, 295, 319

Moore, B., Lake, G., \& Katz, N. 1998, ApJ, 495, 139

Moran, S. M., Miller, N., Treu, T., Ellis, R. S., \& Smith, G. P. 2007, ApJ, 659, 1138
Mori, M., \& Burkert, A. 2000, ApJ, 538, 559

Quilis, V., Moore, B., \& Bower, R., 2000, Science, 288, 1617

Roediger, E., \& Hensler, G. 2005, A\&A, 433, 875

Roediger, E., \& Brüggen, M. 2006, MNRAS, 369, 567

Roediger, E., \& Brüggen, M. 2007, MNRAS, 380, 1399

Roediger, E., Brüggen, M., \& Hoeft, M. 2006, MNRAS, 371, 609

Rubin, V. C., Waterman, A. H., \& Kenney, J. D. P. 1999, AJ, 118, 236

Schindler, S., \& Diaferio, A. 2008, [arXiv:0801.1061]

Schulz, S., \& Struck, C. 2001, MNRAS, 328, 185

Springel, V. 2005, MNRAS, 364, 1105

Springel, V., \& Hernquist, L. 2003, MNRAS, 333, 649

Springel, V., Di Matteo, T., \& Hernquist, L. 2005, MNRAS, 361, 776

Toniazzo, T., \& Schindler, S. 2001, MNRAS, 325, 509

Vollmer, B. 2003, A\&A, 398, 525

Vollmer, B., Marcelin, M., Amram, P., et al. 2000, A\&A, 364, 532

Vollmer, B., Cayatte, V., Balkowski, C., \& Duschl, W. J. 2001, ApJ, 561, 708

Vollmer, B., Balkowski, C., Cayatte, V., van Driel, W., \& Huchtmeier, W. 2004, A\&A, 419, 35

Vollmer, B., Soida, M., Chung, A., et al. 2008, A\&A, 483, 89

Weiner, B. J., Willmer, C. N. A., Faber, S. M., et al. 2006, ApJ, 653, 1049

Ziegler, B. L., Böhm, A., Fricke, K. J., et al. 2002, ApJ, 564, L69

Ziegler, B. L., Böhm, A., Jäger, K., Heidt, J., \& Möllenhoff, C. 2003, ApJ, 598, L87

Ziegler, B. L., Kutdemir, E., Böhm, A., et al. 2007, IAU Symp., 235, 258 